\title{
The evolution of neuroimaging of spinal cord injury patients over the last decade
}

\author{
M Perovitch MD, H Wang MD, S Perl MD \\ The Johns Hopkins University Medical Institutions, Baltimore, and MRI Laboratories, \\ Clinton, Maryland, USA.
}

\begin{abstract}
A review is presented concerning the development of new neuroimaging techniques in the last decade which have improved the diagnostic exploration of patients with spinal cord injuries, including studies of possible sequelae. A number of technical developments occurred in the 1980s, which have broadened the diagnostic capabilities of neuroimaging and made its investigative techniques more precise and less invasive. In a summarized way the new or upgraded modalities such as magnetic resonance imaging and angiography, computed tomography and ultrasound, are considered here with regard to the effect that they have had on the clinical management of patients with a traumatized spinal cord.
\end{abstract}

Key words: neuroimaging advancements; spinal cord trauma.

\section{Introduction}

Neuroimaging has undergone more changes in the 1980s than during the previous 30 years. Many of these changes resulted from the spectacular advances in biomedical engineering and computer science, especially in microcomputerization, general miniaturization of electronic and mechanical equipment, which have all helped neuroimaging to provide more precise anatomical and physiological information. The extensive role of neuroimaging in the 1980 s in disease diagnosis and therapy could not have been anticipated a decade ago. Nor could the concept of imaging the central nervous system without ionizing radiation have been predicted. The tendency to make neuroimaging procedures non invasive or less invasive is a significant change which has occurred in the last decade and is expected to continue in the next one.

From among the many neuroimaging technical developments that occurred in the 1980s, we have selected those that have specific interest for the imaging of a traumatized spinal cord to be discussed in this review.

Correspondence: 48 High Farms Rd, West Hartford, CT 06107, USA.

\section{Magnetic resonance imaging (MRI)}

MRI was introduced as a new neuroimaging tool in June 1980, and in 1981 there were only a few MRI equipments installed and used for the exploration of the brain and spinal cord (in USA). By the end of the decade there were more than one 1000 . This rapid proliferation of a rather expensive imaging modality, especially in the period when computed tomography (CT) and ultrasound were the dominant neuroimaging techniques, is due to the unique capabilities of MRI. It provides direct imaging of the spinal cord in any plane, as well as a superb soft tissue contrast resolution superior to that of CT, without intrathecal instillation of contrast media. Furthermore, there are no known adverse biological effects associated with MRI. This makes possible the repetition of the MRI as many times as necessary in an acute, subacute or chronic phase of a spinal cord injury, and detecting as well as following up the development of trauma's sequelae. ${ }^{1-}$

In the few years since MRI appeared on the clinical arena, notable imaging improvements have taken place. The scanning speed has decreased from minutes even to realtime scanning. Slice thickness has concurrently been reduced to less than $5 \mathrm{~mm}$. The 
elimination of different artifacts, including those caused by respiratory motion and the introduction of refined coils, have further upgraded the quality and diagnostic precision of MRI of the spinal cord trauma. ${ }^{5-7}$

Among the more recent technical advancements of MRI, 4 are especially significant for the evaluation of spinal cord injuries, and should be mentioned in the following text.

$1 M R$ image enhancement was first achieved by introducing the paramagnetic enhancer gadolinium diethylenetriaminopenta-acetic acid (Gd-DTPA). Despite the high level of contrast displayed in MR images, a further increase of contrast may be needed in some instances in order to be able to recognize and identify the type of a post traumatic spinal cord lesion. This can be achieved either through the introduction of new pulse sequences, or substances that will act as paramagnetic enhancers. The application of gadolinium, in our experience, was particularly rewarding in the evaluation of acute and subacute intramedullary hematoma, as well as of the acute spinal cord contrusion. Also, more accurate assessment of myelomalacia is possible in gadolinium enhanced images. Furthermore, the sequelae of a spinal cord trauma, such as cavities (post traumatic multiloculated syringomyelia) or gliosis, epidural scars and diffuse meningeal adhesions, are more evident in the images obtained following the gadolinium infusion. ${ }^{8-11}$

2 Signal suppression techniques contribute also to the clarity of post traumatic spinal cord lesions in MR images. Namely, the signals originating from the fat tissue in the spinal canal can cause similar contrast intensity as the gadolinium enhanced lesion. Thus, the differentiation of the lesion in the spinal cord from the fatty tissue may be difficult. The use of fat-suppression sequences, without or in conjunction with gadolinium, is usually the answer in these situations. ${ }^{12-15}$

3 Three dimensional MRI (3-D imaging) or volume-acquired imaging represents a major advancement in MR technology. The main advantage of 3-D technique is its capability to collect data from the entire volume of the region under examination instead of a single scan at a time as is the case with the, so far used, 2 dimensional imaging (2-D imaging). In addition, data received can be reformatted so that one can see any segment of the examined region from any angle. The 3-D imaging can provide high resolution images of a pathological process in all dimensions from a single acquisition. This ability shortens the imaging time, thus making the examination much more comfortable for a person with spinal cord trauma. ${ }^{16,17}$

4 Magnetic resonance angiography $(M R A)$ has opened new possibilities for the evaluation of the blood flow in the region of a spinal cord injury. So far, the selective spinal cord angiography (standard or digital subtraction) has been the method of choice to demonstrate the spinal cord blood flow. However, ths procedure is associated with high doses of radiation, complex instrumentation and multiple contrast media injections, all of it making selective angiography hazardous, long lasting and difficult to endure by a post traumatic paraplegic or quadriplegic patient. ${ }^{18,19}$ Contrary to this, MRA is a non invasive diagnostic procedure suitable for the imaging of the spinal cord blood circulation, with or without gadolinium enhancement and usually in conjunction with the 3-D MRI. It is safe, and it causes little discomfort to the patient. Nevertheless, at present the experiences with MRA of spinal cord trauma are rather limited, ${ }^{17}$ but its broader use in the evaluation of blood flow in a traumatized spinal cord is only a question of time. ${ }^{20-22}$

\section{Computed tomography (CT)}

In the 1980s CT reached a high level of technical sophistication. The technical improvements relevant to the examination of spinal trauma include thin sections with 
upgraded resolution and diagnostic accuracy, 3-dimensional imaging, decreased reconstruction time, and myelography combined with high resolution CT. Concerning myelography one should add that since metrizamide, the first nonionic contrast medium, became available for intrathecal instillation it has virtually replaced pantopaque and similar contrast agents. The new low-osmolar contrast media appeared in the last decade. Like the nonionic metrizamide, these new contrast agents had an osmolality about half that of former agents with the same iodine content. They share with conventional contrast media the equal stability in solutions, but cost 15 to 20 times more. While the new low-osmolar agents appear superior in a certain way, their high cost relative to medical advantage has led to limited utilization. However, the fast development of MRI in the 80s has overshadowed the advances of CT technology and reduced the use of metrizamide myelography.

\section{Ultrasound}

In the clinical setting ultrasound evolved slowly during the 1960 s and 70 s, although its non invasive nature, rather low cost, easy displacement and safety made this diagnostic modality attractive. In the 1980s a rapid development of ultrasound technology occurred resulting in great improvement of image quality, introduction of color Doppler and development of specialized transducers. The remarkable improvement of image quality was due to the application of computer technology. These changes made ultrasound more competitive with other neuroimaging methodologies, such as computed tomography, digital subtraction angiography and magnetic resonance imaging. Its usefulness in the evaluation of spinal cord injuries progressed in the past decade, especially when used as an adjunct to the neurosurgical treatment of trauma's sequelae. However, as is the case with CT, the diagnostic significance of ultrasound for the exploration of spinal cord injuries is overshadowed by MRI.

\section{Positron emission tomography (PET) and single-proton emission computed tomography (SPECT)}

Both PET and SPECT became accepted as clinical modalities in the 1980 s. PET's ability to image the chemistry of the brain is the subject of many intensive studies. It is obvious that PET, and more recently SPECT, can provide meaningful information about the brain's functioning under normal and pathological conditions. For example, the measurements of brain glucose metabolism has fostered the ability to examine the process of neurotransmission. However, despite the progress made in the better understanding of some brain functions, the application of the 2 modalities in the clinical evaluation of the chemistry in the spinal cord injured tissue has not shown expected results in the 1980 s. The high cost of such studies and the present scarceness of expensive equipment may be additional hindering factors. ${ }^{23}$

\section{Magnetic resonance spectroscopy (MRS)}

MRS has made rather slow progress in the 1980 s as a clinically useful modality. While the number of clinical MR spectroscopic studies of the brain pathology has increased with the availability of the new instrumentation, still many problems obstruct its full utilization. The clinical application of MRS as a modality for the chemical analyses of the intramedullary pathology, and especially traumatology, has not been realized in the 1980s. Although, MRS has great potential, it remained, in the last decade, in a transitional stage between experimental and clinical setting. ${ }^{24,25}$

\section{Conclusion}

A rapid proliferation of complex and sophisticated medical technology in the 1980 s also had an impact on neuroimaging. Due to this, fundamental changes occurred in neuroimaging instrumentation in the last decade, improving the diagnostic quality and capability of its procedures. New concepts concerning neuroimaging emerged, 
such as the need to introduce new equipment that would be safer reducing or eliminating the ionizing radiation and instrumentation. New non-invasive modalities replaced the previous equipment, for example the highly specialized unit for pneumoencephalography. The 1980s were the decade of previously unseen expansion of neuroimaging technology which specifically affected the exploration of spinal cord injuries.

\section{References}

1 Bradley WGF, Walush V, Yalley RA, Wyecof RR (1984) Comparison of CT and MR in 400 patients with suspected disease of the brain and cervical spinal cord. Radiology 152: 695-702.

2 Kneeland JB, Knowles RJR, Cahill PT (1984) Magnetic resonance imaging systems: optimization in clinical use. Radiology 153: 473-478.

3 Perovitch M. Advancements in the neuroimaging of the spinal cord (1987). Paraplegia 25: 244-249.

4 Perovitch M (1985) Assessment of recent neurological imaging modalities. XIIth World Congress of Neurology, Hamburg: Springer International 232 (Suppl) 1-306; 5.

5 Chakers WD, Flickinger F, Bresnahan CJ et al. MR imaging of the acute spinal cord trauma. AJNR 8: 5-10.

6 Wehrli FW, MacFall JB, Shutts D et al (1984) Mechanisms of contrast in NMR imaging. J Comput Assist Tomogr 1984: 8: 369-380.

7 Perovitch M (1987) The application of magnetic resonance in spinal cord disorders. Paraplegia 25: $373-380$.

8 Brasch RC (1983) Methods of contrast enhancement of NMR imaging and potential application. Radiology 147: $781-788$.

9 Perovitch M (1986) The role of 'contrast media' in MR imaging. In: Reisner T, Binder H, Deisenhammer E. eds. Advances in Neuroimaging. Verlag der Wiener Medizinische Akademie, Wien: 356-359.

10 Valk J (1988) Gd-DTPA in MR in spinal lesions. AJR 150: 1161-1168.

11 Breger KR, Williams LA, Daniels LD et al. Contrast enhancement in spinal MR imaging. AJNR 10: 633-637.

12 Hore PJ (1983) Solvent suppression in fourier transform nuclear magnetic resonance. J Magn Reson 55: 283-300.

13 Szumowski J. Plewes DB (1987) Separation of lipid and water MR imaging signals by chopper averaging in the time domain. Radiology 165: 247-250.

14 Perovitch M, Wang H, Perl S (in press) La pathologie des nerfs craniens dans les images de la résonance magnetique. Meeting of the Swiss Societies of Neurology and Neurosurgery. Locarno 1990.

15 Perovitch M, Perl S, Wang H (1991) New MRI methods used in diagnosing unusual multiple sclerosis lesions. Proceedings of the 7th Congress of the European Committee for Treatment and Research in Multiple Sclerosis, St. Gallen: 10.

16 Lang P, Genant HK, Steiger P, et al (1989) 3-D reformatting asserts clinical potential in MRI. Diagn Imaging 11: 80-84.

17 Perovitch M, Bryan RN, Glickson JD, et al (1990) Advancements in the MR imaging and spectroscopy of the central nervous system neoplasms. Invited paper, XIV World Congress of Neurology, New Delhi 1989. In: Gerstenbrand F. ed Advances in Clinical Neuroimaging. Elsevier Science Publishers, Amsterdam-New York: 73.

18 Perovitch M (1981) Spinal cord angiography: trauma of the spinal cord. In: Perovitch M, ed. Radiological Evaluation of the Spinal Cord, Vol. II. CRC Press, Boca Raton, Florida: 84.

19 Perovitch M (1983) Neuroradiology of the sequelae of the spinal cord trauma. In: Adams JH ed. Trauma and regeneration. Acta Neurohirurgica (Suppl 32). Springer Verlag, New York, Wien: 91.

20 Dormont D, Gelbert F, Assouline E et al (1988) MR imaging of spinal cord arteriovenous malformations at 0,5 T: study of 34 cases. AJNR 9: $833-838$.

21 Egerter DE (1991) X-ray angio declines as MRA use expands. Diagn Imaging February: 131-142.

22 Edelman RR (1991) Advances roll back limitations of MRA. Diagn Imaging February: 119-127.

23 Marrett S, Evans AC, Collins I et al (1988) Three-dimensional MR-PET imaging in the human brain. Radiology (Suppl): 169-369.

24 Perovitch M, Bryan RN, Glickson JD, Perl S, Kumar AJ, Wang H (1991) Comparative study of MR imaging and spectroscopy and pathological findings in some brain neoplasms. In: Sinha KK, Chandra P, eds. Progress in Clinical Neurosciences. Vol. 1. Catholic Press, Ranchi, India: 199.

25 Perovitch M, Bryan RN, Glickson JD et al (in press) Evaluation of CNS tumors by means of new MRI techniques. In: Proceedings XIth International Congress of Neuropathology. Kyoto 1990: 277. Jap J Neuropathol (Suppl). 